\title{
Research on microwave mode and coupling energy in plasma reactor design for MPCVD diamond
}

\author{
XiaoJing Li ${ }^{1, a^{*}}$, ShunQi Zheng ${ }^{1, b}$, Hongwei Liu ${ }^{1, c}$, Chunyu Gao ${ }^{2, d}$, \\ Xiumei Shi ${ }^{1}$, Qingxiao $\mathrm{Li}^{1, \mathrm{e}}$, Yang $\mathrm{Ni}^{1}$, Yong $\mathrm{He}^{1}$ \\ ${ }^{1}$ The Ningbo branch of Ordnance Science institute of China, Ningbo 315103, China \\ ${ }^{2}$ The Research Center of State Administration of Work Safety, Beijing 100713, China \\ aemail:happybuaa@126.com , ’email:nbkjhz@163.com, cemail: liuliuhongwei@139.com, \\ demail: gaochunyu@ccsr.cn eemail:cheerxx@126.com
}

\begin{abstract}
Keywords: Microwave plasma chemical vapor deposition (MPCVD), Plasma reactor, Coupling efficiency, Electric field distribution
\end{abstract}

\begin{abstract}
Systematic research on the performance of former designed plasma reactor was carried out in this paper. Numerical simulation method was performed, the distribution of electromagnetic field in microwave cavity by Finite Element Method (FEM). Microwave mode in waveguide, coaxial transformation part and plasma reactor were studied, they are $\mathrm{TE}_{10}$, TEM and $\mathrm{TM}_{021}$ respectively. The impact factor of microwave coupling efficiency was studied. The research results show that, the shape of interconnection between the inner conductor of the antenna and the substrate holder has influence on microwave coupling efficiency. The coupling energy is different when the radius of coaxial conductor was changed, but with the same microwave resonate mode inside the cavity. The research results will provide theory basis for the improvement of device for diamond film deposition, it is also very meaningful for the development of MPCVD technique.
\end{abstract}

\section{Introduction}

Diamond films possess a number of outstanding physical properties, including extreme hardness, high thermal conductivity and wide-band optical transparency. This makes diamond films attractive for a large number of applications. Among the various techniques to deposit diamond films, microwave plasma chemical vapor deposition (MPCVD) has gained more attention due to several reasons: no pollution from electrodes, high density of the plasma, well controlled microstructures as well as high quality of deposited diamond films.

The plasma reactor is the most important component of MPCVD equipment. Design and improvement of the plasma reactor are the key issue for the development of the MPCVD techniques. The coupling structure of plasma reactor will have the ability of focusing more microwave energy. It's meaningful to increase microwave input power and the deposition rate of film.

Several different types of energy coupling mode between microwave and plasma in MPCVD system, such as surface wave coupling type, direct coupling and antenna coupling type [1]. Lower microwave power is allowed to enter the plasma reactor for both surface wave coupling and direct-coupling type MPCVD device. Using antenna coupling ways, the process of microwave coupling and plasma forming to be realized in the two isolated area respectively, which helps to 
enter more energy and less pollution caused by quartz tube or quartz window. At present, antenna coupling way are widely adopted in the MPCVD diamond film deposition equipment[2-4] New MPCVD plasma reactor with coaxial antenna can both as a coupling structure and support substrate holder was studied[5, 6 ].

Deposition of diamond thin film/coating with high speed, it needs high plasma density in plasma reactor. For this reason, the design of microwave resonant cavity, the electric field coupled into the cavity hope to maximize value, in favor of the excitation of the plasma. During the process of MPCVD device design, how to improve the microwave coupling efficiency, and obtain more microwave energy that used for depositing film, is always an important issue to solve.

\section{Numerical simulation methods}

The numerical simulation work has been conducted by the Finite Element Method (FEM). Microwave mode and coupling efficiency were obtained by modeling electric field distribution inside MPCVD device [7]. The governing Maxwell's equation was solved, as the equation (1) and equation (2) shown. Boundary conditions for electric fields on surfaces of metallic components are such that the tangential component of the electric field is zero [8]. A driven-model was employed assuming that a microwave was fed into the cavity through the coaxial antenna. The frequency of the input microwave is $2.45 \mathrm{GHz}$, and at this stage of the simulation, no plasma was considered.

Maxwell's equation:

$$
\nabla \times E=-\mu \frac{\partial H}{\partial t}
$$

$$
\nabla \times H=\varepsilon \frac{\partial E}{\partial t}+J
$$

Here, $E$ is electric field, and $H$ is magnetic field.

\section{MPCVD system with new plasma reactor}

Fig.1 (a) shows schematically the cross-sectional view of MPCVD setup with the proposed reactor, It has been reported in the previous works[6]. The designed plasma reactor used for thin film deposition is the most important part of the device. The deposition system including microwave power, microwave transmission and transformation parts, piping for cooling water and working gas. Short-circuiting piston, adjusting mechanism and three stub tuner are used for adjustment of reflection during the deposition process. 

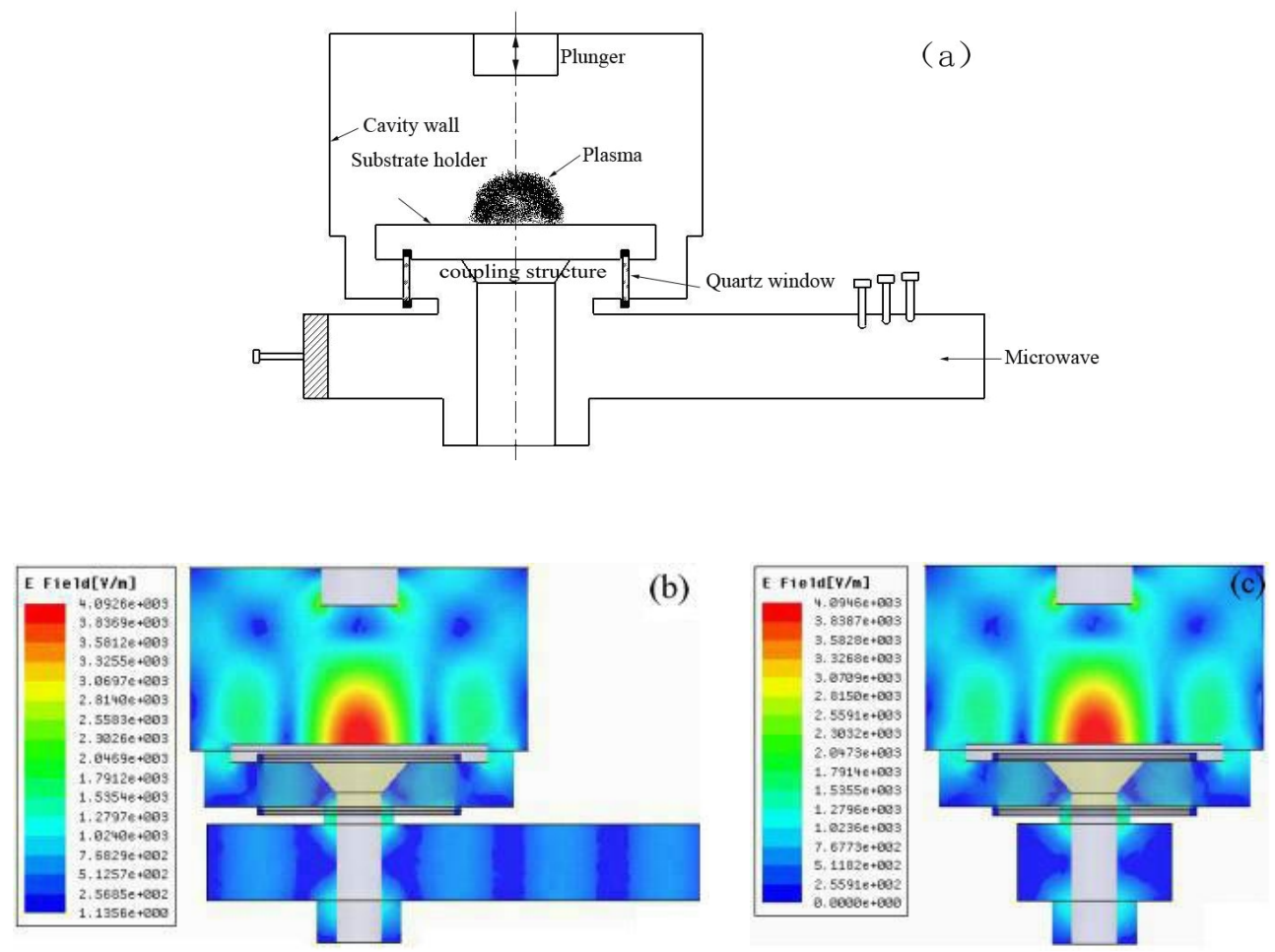

Fig.1 The simulation results of optimization for the whole MPCVD equipment

(a) The cross-sectional view of MPCVD setup

(b) Electric field distribution of the YOZ plane (c) Electric field distribution of the XOY plane

The whole microwave plasma chemical vapor deposition system was analyzed by numerical simulation, which including microwave transmission part, coaxial conversion and microwave cavity. The results can give a dynamic demonstration of the microwave conversion and resonance process. The electric field distribution of the whole deposition system after optimization was shown in Fig.1(b) and Fig.1(c). As Fig.1 (b) and (c) illustrated, the distribution of electric field are symmetrical both on YOZ and XOZ plane. It will ensure that uniform diamond film could be obtained during the MPCVD process.

\section{Microwave transmission mode}

Microwave mode in waveguide and coaxial transformation part were studied. Transmission of microwave is under going through rectangular waveguide, the microwave transmission mode is $\mathrm{TE}_{10}$, as Fig.2 (a) (top view) and Fig.2 (b) (side view) shown. After conversion by the coaxial part, the resonance mode is converted to the axis-symmetric TEM mode, as Fig.3(a) and Fig.3(b) shown. Fig.3(a) shows the distribution of electric field in the cross-section drawn of microwave mode in coaxial part (between inner and outer conductor), while Fig.3(b) demonstrates electric field near the interconnection between the inner conductor and the substrate holder, they are symmetrical. Meanwhile, microwave will be coupled into plasma reactor (resonance cavity), the pattern is $\mathrm{TM}_{021}$, as Fig.1(b) and Fig.1 (c) shown. 

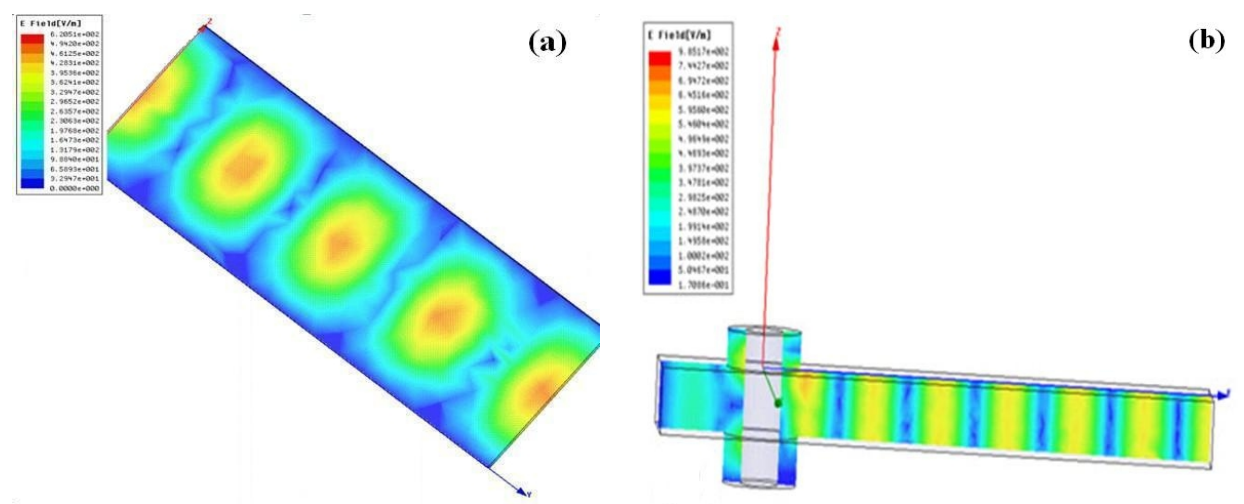

Fig.2 Microwave mode in waveguide $\left(\mathrm{TE}_{10}\right)$

(a)Top view (b) Side view
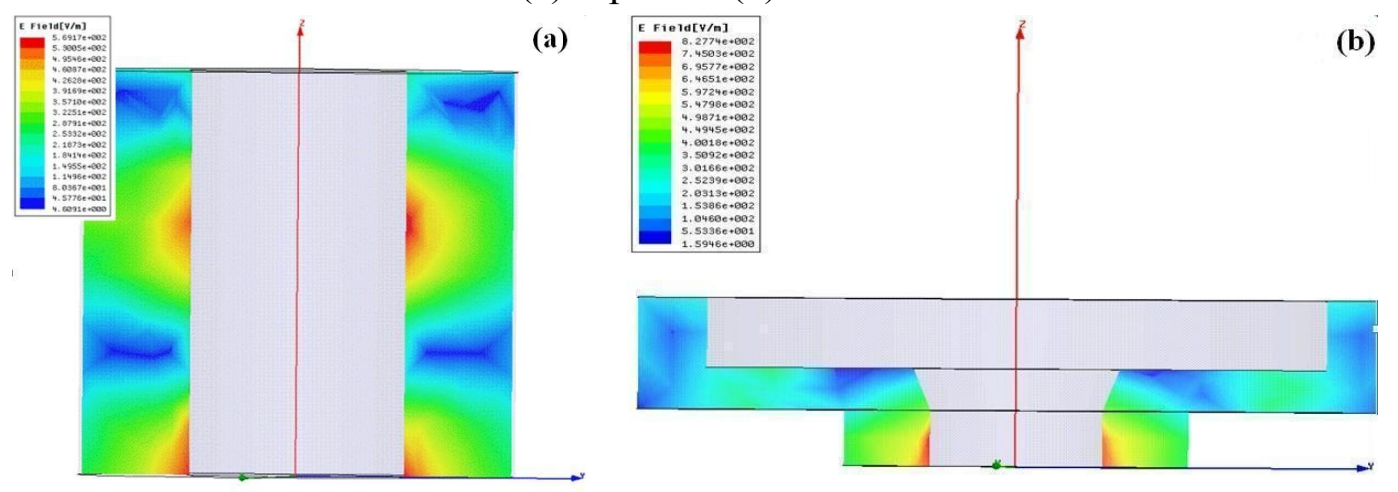

Fig.3 Cross-section drawn of microwave mode in coaxial transformation part (TEM) (a) Coaxial part (b) Interconnection between the inner conductor and the substrate holder

\section{Analysis of coupling efficiency(energy) of microwave power}

It is well known that the geometry of a MPCVD reactor has a great influence on the distribution of electric field inside the reactor. During the optimization, all components of the reactor, including the main chamber, the plunger, the substrate holder, the coaxial antenna as well as the quartz window were modified, so as to maximize the coupling efficiency of microwave power into the chamber.

As far as coupling structure, coupling efficiency of microwave transmission is an important issue. The design rule for coupling structure is that, try to make more microwave power transfer through it and then entry into the cavity, at the same time with the lowest attenuation, in order to avoid energy loss. The influence of coaxial line on coupling energy was study firstly. coupling energy has relations with characteristic impedance of the coaxial line, which can be expressed as $Z_{0}$, as the equation (3) shown[8] :

$$
\mathrm{Z}_{0}=\frac{U}{I}=\frac{60}{\sqrt{\varepsilon_{r}}} \ln \frac{b}{a}(\Omega)
$$

here $b$ and $a$ is the radius of the coaxial conductor separately, $\varepsilon_{r}$ is the relative dielectric 
constant of filling medium in the coaxial line .

According to reference [8], more energy could be transfer into the cavity, the coupling structure will has higher coupling efficiency, when the ratio of two coaxial radius (inner and outer) $\frac{b}{a}=1.65$, the characteristic impedance of coaxial line is $30 \Omega$.At this point, $\mathrm{b}=50 \mathrm{~mm}, \mathrm{a}=$ $30 \mathrm{~mm}$. In the case of fixed diameter of outer conductor, coaxial line won the lowest attenuation coefficient, as the value $\frac{b}{a}=3.59$, here, $b=50 \mathrm{~mm}, a=14 \mathrm{~mm}$, characteristic impedance is 77 $\Omega$. During the process of optimization, $b$ was given a fixed value, the diameter of inner conductor varied from 14 to $30 \mathrm{~mm}$, the maximum electric field strength within the cavity can be obtained as shown in Fig.4(a). It can be seen from optimization results that, the electric field intensity varied with the radius of the coaxial, but with the same microwave resonate mode inside the cavity. Fig. 4 (a) shows the maximum electric field intensity on the center of the substrate holder vary with the radius of inner conductor $a$, it means the coupling energy is different, when the coupling structure with different size.

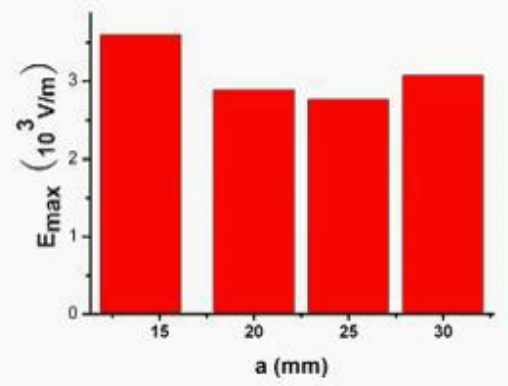

(a)
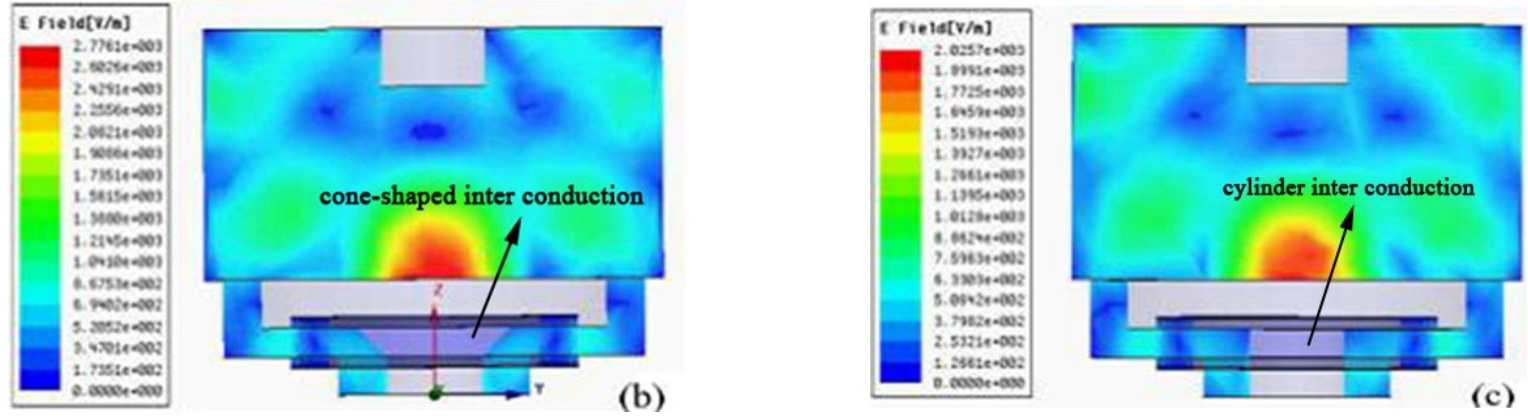

Fig.4 Electric field intensity and distribution simulated for a reactor

(a) The maximum electric field intensity within the cavity vary with the radius of inner conductor

(b) With cone-shaped (c) with cylinder inter conduction between antenna and substrate holder

The interconnection between the inner conductor of the antenna and the substrate holder was also optimized. Fig.4(b) and Fig.4(c) shows a comparison of representative simulation results of the reactor, with and without a cone-shaped interconnection. It can be found from this figure that with the cone-shaped interconnection the maximum electric field could reach a value of $\mathrm{E}_{\max }=2.776 \times 10^{4}$ $\mathrm{V} / \mathrm{m}$ compared with a value of $\mathrm{E}_{\max }=2.025 \times 10^{4} \mathrm{~V} / \mathrm{m}$ when this cone-shaped interconnection was absent ,and only cylinder shape inter conduction was used . 
In addition, during the process of design coupling structure, their mechanical properties for supporting function and processing performance should be considered. On the one hand, if it is designed with small size, it not only couldn't support the substrate holder well but also impede cooling effect. On the other hand, coupling structure with big size has bad processing performance, it's not easy to manufacture and assemble. In one word, the radius of coaxial conductor is neither too large nor too small, so it is very essential to find the right size by numerical simulation.

\section{Conclusions}

Based on numerical simulation, systematic theoretic study on the performance of a new designed microwave plasma reactor with simple geometric structure was carried out. Microwave mode in waveguide, coaxial transformation part and plasma reactor were studied. They are TE10, TEM and TM021 respectively. The research results show that, coupling structure has influence on the microwave input power and coupling efficiency of the cavity. During the process of design coupling structure for microwave transmission, three essentials should be considered, they are: (1) mechanical properties, supporting effect (2) processing performance, easy to manufacture (3) coupling efficiency, aim to avoid energy loss during the process of microwave transmission.

\section{Acknowledgements}

This work is financially supported by the Natural Science Foundation of Zhejiang province grant No. LQ13F010002. Public Project of Zhejiang Province under Grant No. 2014C31113. Natural Science Foundation of Ningbo under Grant No. 2014A610012. Priority setting for postdoctoral research Project of Zhejiang Province under Grant No. BSH1302058.

\section{References}

[1] Huang Jianliang, Wang Jianhua, Man Weidong. The development of Apparatus to grow microwave plasma chemical vapor deposition diamond films.Vacuum \& Cryogenics.2008, 14(1): $1-5$.

[2] Fuener M, Wild C, Koidl P. Novel microwave plasma reactor for diamond synthesis. Applied Physics Letters, 1998, 72 (10): 1149-1151.

[3]Pleuler E, Wild C, M. Funer, et al. The CAP-reactor, a novel microwave CVD system for diamond deposition. Diamond and Related Materials, 2002, 11: 467-471.

[4] Silva F, Hassouni K, Bonnin X, et al. Microwave engineering of plasma-assisted CVD reactors for diamond deposition. Journal of Physics: Condensed Matter, 2009, 21: 1-16.

[5] A. Kromka, O. Babchenko, T. Izak, K. Hruska, B. Rezek Linear antenna microwave plasma CVD deposition of diamond films over large areas .Vacuum .2012,86: 776-779.

[6] X.J. Li, W.Z. Tang, S.W. Yu, S.K. Zhang, G.C. Chen, F.X. Lu, Design of novel plasma reactor for diamond film deposition, Diamond and Related Materials,2011, 20 :480. 
[7] Y.J. Xie, P. Wang, L. Li. Basic and application of Ansoft HFSS, Xi'an University of Electronics Science and Technology Press, Xi 'an, 2007. (In Chinese)

[8] C.H. Zhao, Z.Z. Zhang. Microwave Technology, Higher Education Press, Beijing, 2007. (In Chinese) 